\title{
Direct visualization of an antidepressant analog using surface-enhanced Raman scattering in the brain
}

\author{
Masato Tanuma, ${ }^{1}$ Atsushi Kasai, ${ }^{1}$ Kazuki Bando, ${ }^{2,3}$ Naoyuki Kotoku, ${ }^{4}$ Kazuo Harada, ${ }^{5,6}$ \\ Masafumi Minoshima, ${ }^{7}$ Kosuke Higashino, ${ }^{1}$ Atsushi Kimishima, ${ }^{8}$ Masayoshi Arai, ${ }^{8}$ \\ Yukio Ago, ${ }^{1,9}$ Kaoru Seiriki, ${ }^{1,10}$ Kazuya Kikuchi, ${ }^{7,11}$ Satoshi Kawata, ${ }^{2,3}$ Katsumasa Fujita, ${ }^{2,12,13}$ \\ and Hitoshi Hashimoto ${ }^{1,12,14,15,16}$
}

'Laboratory of Molecular Neuropharmacology, Graduate School of Pharmaceutical Sciences, and 'Department of Applied Physics, Graduate School of Engineering, Osaka University, Suita, Osaka, Japan. ${ }^{3}$ Serendip Research, Osaka, Osaka, Japan. ${ }^{4}$ Chemical Biology Laboratory, College of Pharmaceutical Sciences, Ritsumeikan University, Kusatsu, Shiga, Japan. ${ }^{5}$ Department of Legal Medicine, Graduate School of Medicine, ${ }^{6}$ Laboratory of Applied Environmental Biology, Graduate School of Pharmaceutical Sciences, ${ }^{7}$ Laboratory of Chemical Biology, Graduate School of Engineering, ${ }^{8}$ Laboratory of

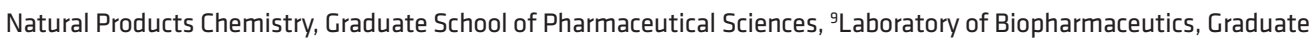
School of Pharmaceutical Sciences, ${ }^{10}$ Institute for Transdisciplinary Graduate Degree Programs, ${ }^{11}$ Immunology Frontier Research Center, and ${ }^{12}$ Institute for Open and Transdisciplinary Research Initiatives, Osaka University, Suita, Osaka, Japan. ${ }^{13}$ Advanced Photonics and Biosensing Open Innovation Laboratory, AIST-Osaka University, Suita, Osaka, Japan. ${ }^{14}$ Molecular Research Center for Children's Mental Development, United Graduate School of Child Development, Osaka University, Kanazawa University, Hamamatsu University School of Medicine, Chiba University and University of Fukui, Suita, Osaka, Japan. ${ }^{15}$ Institute for Datability Science and ${ }^{16}$ Department of Molecular Pharmaceutical Sciences, Graduate School of Medicine, Osaka University, Suita, Osaka, Japan.

Detailed spatial information of low-molecular weight compound distribution, especially in the brain, is crucial to understanding their mechanism of actions. Imaging techniques that can directly visualize drugs in the brain at a high resolution will complement existing tools for drug distribution analysis. Here, we performed surface-enhanced Raman scattering (SERS) imaging using a bioorthogonal alkyne tag to visualize drugs directly in situ at a high resolution. Focusing on the selective serotonin reuptake inhibitor S-citalopram (S-Cit), which possesses a nitrile group, we substituted an alkynyl group into its structure and synthesized alkynylated S-Cit (Alk-S-Cit). The brain transitivity and the serotonin reuptake inhibition of Alk-S-Cit were not significantly different as compared with S-Cit. Alk-S-Cit was visualized in the coronal mouse brain section using SERS imaging with silver nanoparticles. Furthermore, SERS imaging combined with fluorescence microscopy allowed Alk-S-Cit to be visualized in the adjacent neuronal membranes, as well as in the brain vessel and parenchyma. Therefore, our multimodal imaging technique is an effective method for detecting low-molecular weight compounds in their original tissue environment and can potentially offer additional information regarding the precise spatial distribution of such drugs.

Authorship note: MT, A. Kasai, and KB contributed equally to this work.

Conflict of interest: The authors have declared that no conflict of interest exists.

Copyright: (c) 2020, American Society for Clinical Investigation.

Submitted: September 9, 2019

Accepted: February 19, 2020

Published: March 26, 2020.

Reference information: JCI Insight. 2020;5(6):e133348.

https://doi.org/10.1172/jci.

insight.133348.

\section{Introduction}

Determining the tissue distribution of drugs at their intended target is essential to understanding how these drugs exert their effects. The brain is a complex organ and is anatomically defined into numerous regions that regulate distinct functions $(1,2)$, making it even more important to understand the tissue distribution of drugs such as antidepressants that act on the CNS. For determining the drug distribution in the brain, imaging techniques are considered to be powerful tools.

The tissue distribution of CNS drugs such as selective serotonin reuptake inhibitors (SSRIs) is often studied using imaging techniques including PET and mass spectrometry imaging (MSI). PET employs radioisotopes to label low-molecular weight compounds, and this can be utilized in humans. Previous human studies have revealed a higher S-citalopram (S-Cit) occupancy of the serotonin transporter (SERT) in the raphe nuclei 
and frontal cortex, as compared with the hippocampus and putamen, although at a resolution on the millimeter order (3-5). Alternatively, MSI techniques coupled with matrix-assisted laser desorption/ionization or desorption electrospray ionization (DESI) allow for the direct determination of nonlabeled drug distributions at improved resolutions (6). The DESI-MSI analysis of a single dose of fluvoxamine showed high concentrations in the ventral striatum, thalamus, cerebellum, cortex, caudate putamen, and hippocampus (7). Because the ionization stage of MSI requires the desorption of a molecule from the sample tissue, determining the exact location of a low-molecular weight compound within a designated field of view with respect to the other biological components, such as blood vessels, has remained challenging. A new imaging modality capable of directly imaging low-molecular weight compounds in situ at high resolutions would complement existing techniques and provide an improved outlook on drug distributions.

Spontaneous Raman microscopy can directly image low-molecular weight compounds by detecting the vibrational signals of their specific bonds. However, detecting low-molecular weight compounds at low concentrations remains challenging, owing to the low intensity of the signals from spontaneous Raman scattering (8). Surface-enhanced Raman scattering (SERS) overcomes this problem and amplifies the phenomenon of spontaneous Raman scattering by a factor of $1 \times 10^{4}$ to $1 \times 10^{10}$ based on the adsorption of chemical bonds onto the surfaces of nanoparticles (9-12). A recent report stated that the in vitro detection limit of an alkynylated peptide using SERS was as low as $3.3 \mathrm{nM}$ (13), which would allow for the detection of drugs in tissues. The combination of SERS with fluorescence microscopy is also possible and will allow the in situ observation of alkyne- and fluorescent-labeled substances.

In the present study, we have developed a method for direct visualization of low-molecular weight compounds in the mouse brain at a high resolution through alkyne tag integration and SERS imaging. We synthesized alkyne-possessing S-Cit — namely, alkynylated S-Cit (Alk-S-Cit) — and found that AlkS-Cit displayed similar brain transitivity and functional serotonin reuptake inhibition as compared with S-Cit. SERS imaging successfully detected the alkyne signals from Alk-S-Cit in the brain. Combining SERS imaging with fluorescence microscopy visualized the vessel-parenchyma distributions of Alk-S-Cit. Thus, we were able to directly detect a low-molecular weight compound in the brain. Moreover, the proposed technique can potentially be extended to allow for the elucidation of drug distributions over entire tissues. Our multimodal imaging approach will provide additional information regarding the distribution of lowmolecular weight compounds, which may help to elucidate their underlying mechanism of actions.

\section{Results}

Labeling of S-Cit with alkyne tag. The Raman spectrum of cells and tissues consists of a fingerprint region $\left(<1800 \mathrm{~cm}^{-1}\right)$, with multiple peaks, and a silent region $\left(1800-2800 \mathrm{~cm}^{-1}\right)$, with minimal or no peaks. The alkynyl group $(-\mathrm{C} \equiv \mathrm{CH})$, which is bioorthogonal and rarely found in biological tissues, shows a strong Raman peak in the silent region and can therefore be easily detected $(14,15)$. Furthermore, whereas the application of commonly used fluorophores with low-molecular weight compounds carries the risk of changing the pharmacokinetics and pharmacodynamics of the drugs, the small molecular size of the alkynyl group means it can be appended to these compounds without significantly altering their biological properties (16). Therefore, the alkynyl group has been used as a convenient Raman tag. It has been reported that the cell nuclei in cultured cells were imaged previously by using spontaneous Raman spectroscopy and EdU (5-ethynyl-2'-deoxyuridine), a thymidine analog with an alkynyl group $(14,16)$. To incorporate an alkyne tag within the structure of S-Cit, we focused on the C-5 position of S-Cit for the following reasons (Figure 1A). Firstly, the C-5 position of S-Cit is capable of tolerating the steric bulk of a potentially novel chemical moiety (molecular weight of 200) without a significant loss in the binding affinity of S-Cit to SERT (17-19). The nitrile terminal group $(-\mathrm{C} \equiv \mathrm{N})$ of $\mathrm{S}$-Cit is a moiety that has a similar electronic structure to the alkynyl group $(-\mathrm{C} \equiv \mathrm{CH})$. Using $\mathrm{S}-\mathrm{Cit}$ as the starting material, we modified the nitrile group to an alkynyl group and chemically synthesized Alk-S-Cit (see Methods and Supplemental Figure 1; supplemental material available online with this article; https://doi.org/10.1172/jci.insight.133348DS1). The ${ }^{1} \mathrm{H}$ - and ${ }^{13} \mathrm{C}-\mathrm{NMR}$ spectra and high-resolution electrospray ionization TOF mass spectrometry (ESI-TOF-MS) data $\left(m / z, 324.1764\right.$ calculated for $\mathrm{C}_{21} \mathrm{H}_{23} \mathrm{NOF}$; found, 324.1751) revealed the chemical structure of Alk-S-Cit (Supplemental Figures 2-4).

In silico prediction of binding of Alk-S-Cit to SERT. Any chemical modifications made to low-molecular weight compounds can change their target binding orientation and modify their structure (20). S-Cit primarily acts by binding to SERT and subsequently inhibiting serotonin reuptake (21). To simulate the binding orientation of Alk-S-Cit with respect to SERT and to determine whether it would differ from that of S-Cit, 
A<smiles>CN(C)CCC[C@]1(c2ccc(F)cc2)OCc2cc(S(C)(=O)=O)ccc21</smiles>

S-citalopram

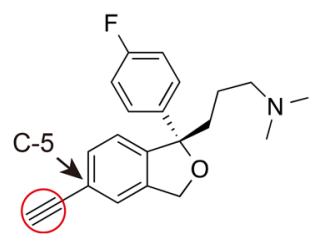

Alkynylated S-citalopram
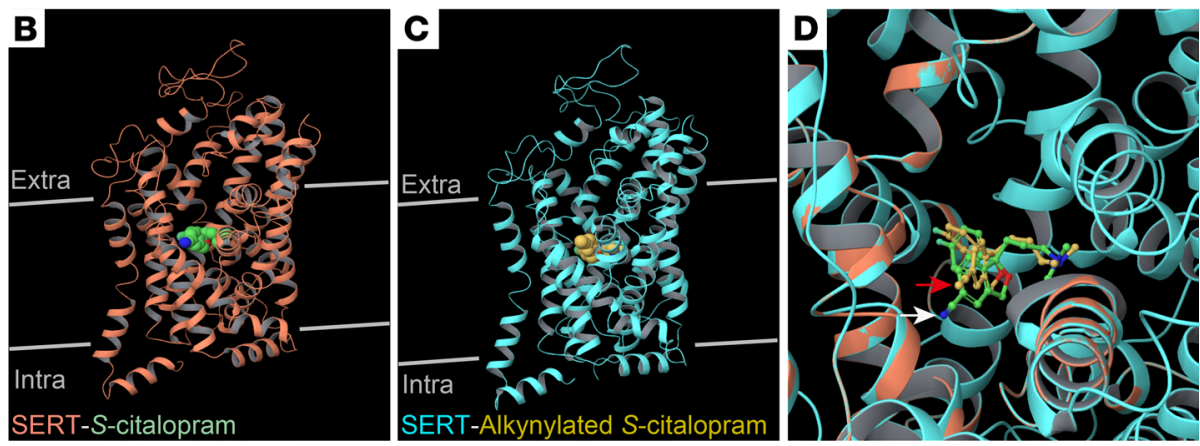

Figure 1. Alkynylated S-citalopram. (A) Structures of selective serotonin reuptake inhibitor analogs. Alkyne tag is shown with a red circle. (B and $\mathbf{C}$ ) In silico prediction of binding of S-Cit (B) and Alk-S-Cit (C) to serotonin transporter. Green and yellow spheres represent carbon atoms; blue, nitrogen atoms; red, oxygen atoms. (D) Magnified view of overlay of binding predictions of $\mathbf{B}$ and $\mathbf{C}$. The white arrow shows the nitrile group of S-Cit. The red arrow shows the alkynyl group of Alk-S-Cit.

we used an in silico modeling method. A recent study elucidated the structure of human SERT (hSERT) and showed how several SSRIs, including S-Cit, bind to its main binding pocket (22). Based on these details of the hSERT protein, we modeled the binding orientation of S-Cit and subsequently replaced its nitrile group with an alkynyl group (Figure 1, B and C). The structures of the surrounding amino acids were optimized to minimize the total energy of the complex structure. Only a minimal change in the binding orientation was observed, which was because the hydrogen atom of the incorporated alkynyl group interacted with the peripheral amino acids (Figure 1, B and C). Next, we compared the binding orientations of the 2 compounds with respect to SERT. The C-5 position of Alk-S-Cit was shifted marginally, compared with that of S-Cit, and was positioned toward the surface of the extracellular membrane (Figure 1D). We observed only small differences in the conformations of the main binding pocket bound with S-Cit or Alk-S-Cit (Figure 1D). These results suggest that Alk-S-Cit would act on the SERT similarly to S-Cit and exhibit SSRI properties.

Brain transitivity of Alk-S-Cit. Although the alkynyl group is a small tag molecule that is thought to have minimal effect on the properties of the incorporating compounds, the incorporation of tags such as fluorophores to low-molecular weight compounds may affect their pharmacokinetics (23). To determine the transitivity of Alk-S-Cit to the brain, we quantified the Alk-S-Cit level in sections of the mouse brain using Liquid chromatography-tandem mass spectrometry (LC-MS/MS) (Figure 2A). The mice were coadministered with $5 \mathrm{mg} / \mathrm{kg}$ doses of S-Cit and Alk-S-Cit, and their brain sections were collected after 1 hour. Previous PET studies have shown that the occupation of SERT by S-Cit is high in the dorsal raphe nucleus and striatum (5). S-Cit and Alk-S-Cit were detected in the homogenate of the $0.5 \mathrm{~mm}$-thick brain sections, including the dorsal raphe nucleus and the striatum, at different retention times (1.04 and 1.44 minutes, respectively; Figure 2, B and C). At 1 hour after the administration of the drugs, there was no significant difference in the levels of S-Cit and Alk-S-Cit in the 2 sections (Figure 2D). These results suggest that the brain transitivity of Alk-S-Cit is similar to that of S-Cit in the 2 regions at the time point tested.

Additionally, we calculated the expected concentration of Alk-S-Cit in the brain by dividing the amount of Alk-S-Cit by the volume of the brain section $\left(0.013 \mathrm{~cm}^{3}\right)$ and found that Alk-S-Cit exists in the nano-molar range $(\sim 420 \mathrm{nM})$.

In vivo analysis of extracellular serotonin after administration of Alk-S-Cit. SSRIs increase the serotonin level in the synaptic cleft by binding to SERT, especially in the medial prefrontal cortex, a brain region implicated in antidepressant effects (24). The medial prefrontal cortex receives inputs from the dorsal raphe nucleus, the largest serotonergic nucleus in the murine brain, and exhibits increased levels of extracellular 
A
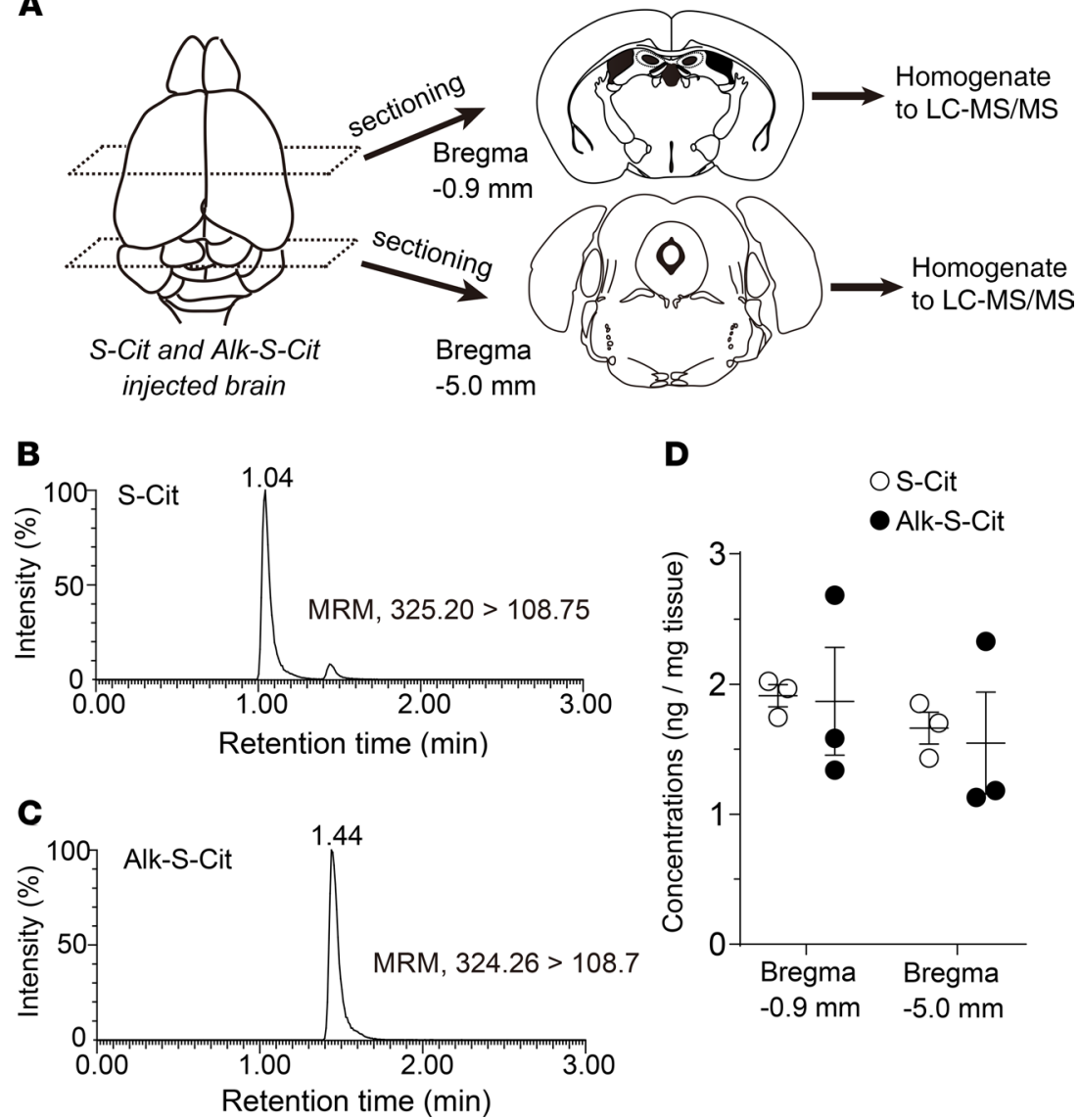

Figure 2. In vivo brain transitivity of Alk-S-Cit. (A) Schematic of determination of chemical concentrations in the brain section. (B and C) Mass chromatograms of S-Cit (B) and Alk-S-Cit (C) in tissue homogenate as determined by LC-MS/ MS multiple reaction monitoring (MRM). (D) Brain concentrations of S-Cit (open circles) and Alk-S-Cit (filled circles) in $5 \mathrm{~mm}$-thick sections (approximately anterior/posterior $-0.9 \mathrm{~mm}$ and $-5.0 \mathrm{~mm}$ from bregma) after combined dose of 5 $\mathrm{mg} / \mathrm{kg}$ dose of each drug $(n=3$ each). Concentrations were measured by LC-MS/MS at 60 minutes after i.p. administration of drugs. Data are mean \pm SEM. Statistical analysis was performed using 2-way ANOVA. Compound effect, $F_{(1,8)}=$ $0.0722, P=0.795$; region effect, $F_{(1,8)}=0.940, P=0.361$; interactions, $F_{(1,8)}=0.0151, P=0.905$.

serotonin after the administration of SSRIs (25). Therefore, to assess the reuptake-inhibiting capability of Alk-S-Cit, we quantified the extracellular serotonin levels by performing in vivo online microdialysis-HPLC on free-moving mice (Figure 3A). The extracellular serotonin levels were recorded for 2 hours at 20-minute intervals after the i.p. administration of either S-Cit or Alk-S-Cit. Alk-S-Cit administration increased the extracellular serotonin levels, which remained high for the total duration of the recording. The increase in extracellular serotonin levels after Alk-S-Cit administration was similar to that after S-Cit administration (group effect, $P>0.05$ ), although we observed a significant group $\times$ time interaction (Figure 3B). These results indicated that the synthesized Alk-S-Cit exhibits strong in vivo serotonin reuptake-inhibiting characteristics at levels comparable with those of S-Cit.

Detection of Alk-S-Cit using SERS. Because Alk-S-Cit was expected to bind to SERT and exhibited brain transitivity and extracellular serotonin release properties similar to those of S-Cit, we assumed that the brain distribution of Alk-S-Cit would be similar to that of S-Cit and attempted to image Alk-S-Cit using SERS. Silver nanoparticles were used for imaging, since they magnify the SERS signal to a greater extent than gold nanoparticles (26). Based on the in silico modeling results (Figure 1C), we expected that nanoparticles with a smaller diameter would be more accessible to the alkynyl group, located at the main binding pocket of SERT. On the other hand, because the SERS signal is weaker when nanoparticles with smaller diameters $(<20 \mathrm{~nm})$ are used (13), we synthesized silver nanoparticles with a diameter of $23 \mathrm{~nm}$ and performed SERS measurements on an Alk-S-Cit solution $(40 \mu \mathrm{M})$ in the presence of these silver nanoparticles on a glass substrate (Figure 4A). Alk-S-Cit showed 2 SERS peaks, at $1990 \mathrm{~cm}^{-1}$ and $2170 \mathrm{~cm}^{-1}$, in the silent 
A

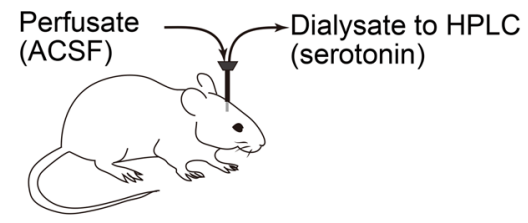

Basal Compound

Cannulation monitoring i.p.

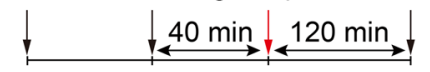

Time course of microdialysis analysis

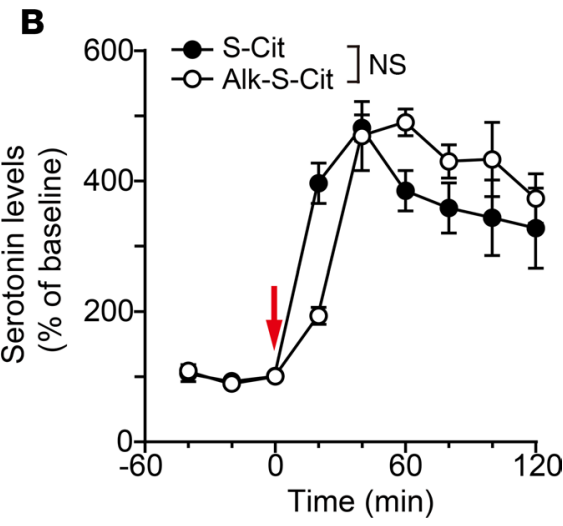

Figure 3. Increase in extracellular serotonin levels after in vivo administration of Alk-S-Cit. (A) Schematic showing monitoring of extracellular serotonin levels in the medial prefrontal cortex by microdialysis and time line of experiments. ACSF, artificial cerebrospinal fluid. Red arrow shows time of compound administration. (B) S-Cit (5 mg/kg, filled circle) or Alk-S-Cit ( $5 \mathrm{mg} / \mathrm{kg}$, open circle) was administered i.p. at 0 minutes (red arrow). Alk-S-Cit induces an increase in serotonin level similar to that of S-Cit in the medial prefrontal cortex. Each point represents 20-minute dialysate sampling period (means \pm SEM percentage of serotonin baseline, $n=3$ for each group). Two-way ANOVA indicates group effect. $F_{(1,36)}=0.4524, P=0.5055$; time effect, $F_{(8,36)}=41.84, P<0.0001$; group $\times$ time interactions, $F_{(8,36)}=3.688$, $P=0.0031$

region and a small spontaneous Raman peak at $2100 \mathrm{~cm}^{-1}$ (Figure 4A). The peak observed at $1990 \mathrm{~cm}^{-1}$ in the SERS spectrum was downshifted compared with the spontaneous Raman peak; this can be attributed to the alkynes adsorbed and bound to the surfaces of the silver nanoparticles. Moreover, the peak observed at a longer wavelength in the SERS spectrum (i.e., $2170 \mathrm{~cm}^{-1}$ ) can be attributed to the alkynes in close proximity but not bound to the surfaces of nanoparticles $(27,28)$.

Next, we tested the sensitivity of the SERS technique by recording the signals from diluted Alk-S-Cit solutions. Strong alkyne SERS signals $\left(2170 \mathrm{~cm}^{-1}\right)$ from a $10-\mu \mathrm{M}$ Alk-S-Cit solution were detected, using glass substrates coated with the synthesized silver nanoparticles (Figure 4B). Conversely, no alkyne signals were observed from the S-Cit solution at the same concentration, confirming that the technique was suitable for the selective detection of Alk-S-Cit (Figure 4B). The alkyne SERS signals showed a concentration-dependent change, and hotspots were detected down to $1 \mathrm{nM}$ on the glass substrate. The probability distributions for SERS signal showed that the charge coupled device (CCD) counts underwent a shift based on the Alk-S-Cit concentration (Figure 4C). Thus, using the silver nanoparticles, we achieved a detection sensitivity at nano-molar levels, sufficient to capture alkyne signals from brain tissues (Figure 2).

We next performed SERS imaging to detect Alk-S-Cit in brain tissues (Figure 4, D-G). A single i.p. dose of Alk-S-Cit was administered to a mouse, whose brain was collected after 1 hour and cut into $20 \mu \mathrm{m}-$ thick sections, which included the dorsal raphe nucleus. A total of $2 \mu \mathrm{L}$ of the silver nanoparticle dispersion was then applied on the surfaces of the tissue sections, which were then immediately covered with a cover glass also coated with the same silver nanoparticle dispersion for the SERS imaging. We examined the averaged SERS images of 500 to $1500 \mathrm{~cm}^{-1}$, which can be attributed to the fingerprint region on the Raman spectrum. Although the intensity of the signals was not uniform, strong SERS signals were observed across the whole field of view, indicating that the distribution of silver nanoparticles was widespread across the entire region of interest (Figure $4 \mathrm{E}$ ). To see whether the silver nanoparticle dispersion had permeated into the tissue section, we performed SERS imaging at 2- $\mu \mathrm{m}$ intervals from the surface of the tissue (Supplemental Figure 5). SERS signals were recorded up to 5 - $\mu \mathrm{m}$ deep into a $20-\mu \mathrm{m}$ brain section. The intensity of SERS was the highest at the surface of the tissue and decreased further into the tissue. Because the pattern of the hotspots were consistent from the surface to the deeper layers, it is likely the signals observed in the deeper layers derived from the tissue surface. Therefore, it is probable that the penetration of silver nanoparticles through the tissue was minimal.

Next, we examined SERS images at approximately $2100 \mathrm{~cm}^{-1}$ for signals derived from the alkynyl group of Alk-S-Cit. Several hotspots were observed from the dorsal raphe of the Alk-S-Cit-administered mouse (Figure $4 \mathrm{~F}$ ). We further examined the SERS spectrum of each observed hotspot and found that each spectrum exhibited a peak at approximately $2170 \mathrm{~cm}^{-1}$ (Figure $4 \mathrm{G}$ ). In contrast, in the dorsal raphe sections of 
A

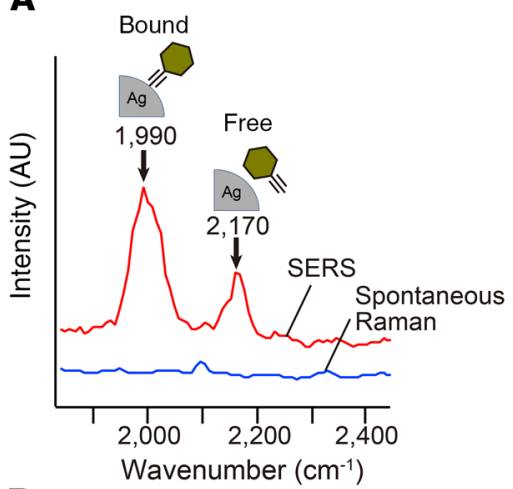

C

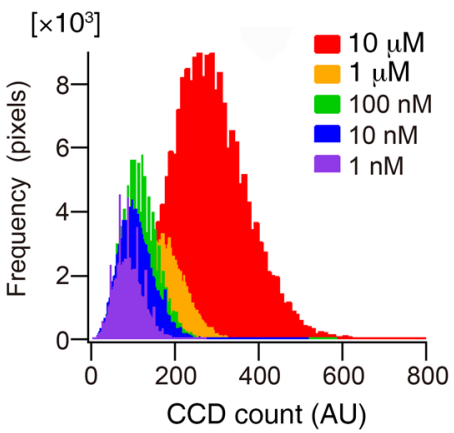

B
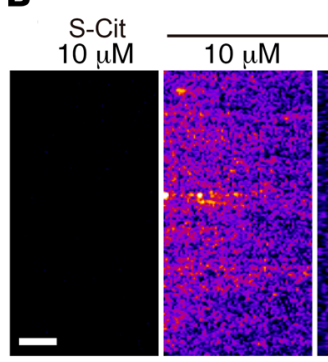

Alk-S-Cit
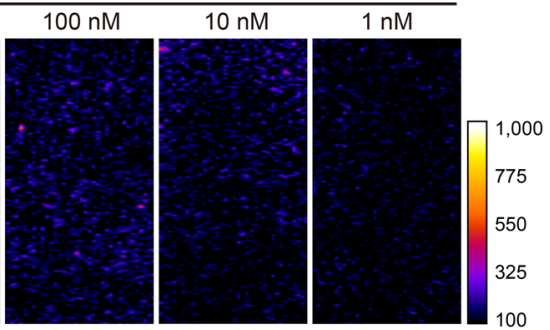

D

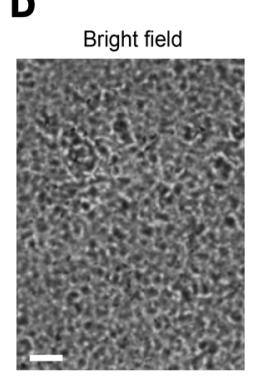

E SERS of fingerprint region
$\mathbf{F}$

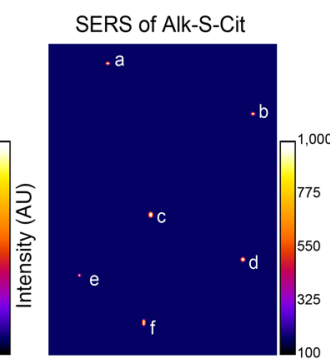

G

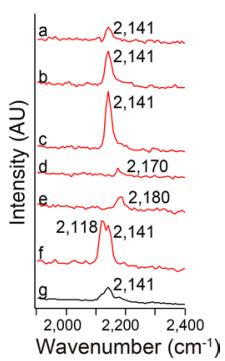

Figure 4. SERS imaging of Alk-S-Cit. (A) Alk-S-Cit solution with concentration of $40 \mu \mathrm{M}$ and exposure time of 2 seconds was used for measurements of spontaneous Raman spectra, while sample solution with a concentration of $40 \mu \mathrm{M}$ and exposure time of 200 ms were used for SERS measurements. The excitation wavelength was $532 \mathrm{~nm}$ and laser power was $0.4 \mathrm{~mW} / \mu \mathrm{m}^{2}$ on sample plane. (B) SERS intensity map at $2170 \mathrm{~cm}^{-1}$ for different concentrations of Alk-S-Cit ( $1 \mathrm{nM}$ to $10 \mu \mathrm{M}$ ) and S-Cit (10 $\mu \mathrm{M})$ measured on glass substrates with dispersed $\mathrm{Ag}$ nanoparticles $(\varphi=23 \mathrm{~nm})$. The excitation wavelength was $532 \mathrm{~nm}$ and laser power was $0.4 \mathrm{~mW} / \mu \mathrm{m}^{2}$ on sample plane. Scale bar: $10 \mu \mathrm{m}$. (C) Probability distributions of SERS intensity for various concentrations of Alk-S-Cit $(1 \mathrm{nM}$ to $10 \mu \mathrm{M})$ are shown as comparative histograms. (D-F) Representative images of bright-field (D), heatmap of SERS signal of fingerprint region (averaged over 500$\left.1500 \mathrm{~cm}^{-1}\right)(\mathbf{E})$, and Alk-S-Cit (averaged over 2141-2180 $\left.\mathrm{cm}^{-1}\right)(\mathbf{F})$ in the same field of view. Scale bar: $10 \mu \mathrm{m}$. (G) Spectrums a-f show SERS spectra at positions shown in $\mathbf{F}$, and spectrum $g$ shows average of these spectra. Each SERS peak $\left(\mathrm{cm}^{-1}\right)$ is shown in $\mathbf{G}$.

the S-Cit-administered mouse, an alkyne-related Raman peak in the silent region was not observed (data not shown). To determine whether SERT expression levels affects Alk-S-Cit distribution, we examined the dorsomedial periaqueductal gray, which expresses low levels of SERT (29). No distinct alkyne peaks were observed from the dorsomedial periaqueductal gray, suggesting a low distribution of Alk-S-Cit to the region (Supplemental Figure 6). Based on the in vitro spectrum of Alk-S-Cit shown in Figure 4, the SERS spectra of the brain tissues, which contained a peaked at approximately $2170 \mathrm{~cm}^{-1}$, confirmed that the detected Alk-SCit would not be bound directly to the silver nanoparticles.

Multimodal imaging by combining SERS and fluorescence microscopy. The SERT is expressed at axons and synaptic terminals of neurons. Multimodal imaging techniques that combine SERS and fluorescence imaging capable of capturing both low-molecular weight compounds and the neuronal membranes will be essential for obtaining critical information concerning the drug target in the brain. To investigate whether the alkyne hotspots would colocalize with axons or synaptic terminals, we simultaneously imaged Alk-S-Cit and fluorescently labeled neuronal axons in brain sections. We administered adeno-associated virus (AAV) expressing Chronos-fused GFP to label neuronal membrane and synaptic terminals (30). GFP was selected because its excitation and emission spectra $\left(\lambda_{\mathrm{ex}}=488 \mathrm{~nm}, \lambda_{\mathrm{em}}=510 \mathrm{~nm}\right)$ do not overlap with the SERS alkyne peaks of the silver nanoparticles used (observed at approximately $601 \mathrm{~nm}$ using a $532 \mathrm{~nm}$ excitation laser). We found 2 alkyne SERS signals in close proximity with neuronal axons in the dorsal raphe (Figure 5, A and B), suggesting the observed Alk-S-Cit were located at potentially SERT-expressing neuronal membranes.

Since the vascular endothelium separates the brain blood vessels from the brain parenchyma, multimodal images capturing both low-molecular weight compounds and the vascular endothelium will be essential for obtaining critical information concerning the brain transitivity of the drugs that target the brain. To this end, we simultaneously imaged Alk-S-Cit and fluorescently labeled blood vessels in mouse 
brain sections. The blood vessels were labeled with an endothelial marker, tomato-lectin (31), conjugated with the fluorophore DyLight $488\left(\lambda_{\mathrm{ex}}=492 \mathrm{~nm}, \lambda_{\mathrm{em}}=520 \mathrm{~nm}\right)$. We observed several alkyne hotspots on the same surface plane as the DyLight 488-labeled blood vessels in the dorsal raphe nucleus (Figure 5C). Most of the hotspots were located in the brain parenchyma region (Figure 5, D-F). Thus, by combining alkyne-based SERS imaging with fluorescence microscopy, we could successfully image the vessel-parenchyma distributions of Alk-S-Cit in situ.

\section{Discussion}

We have developed an imaging approach for directly visualizing low-molecular weight compounds in the brain tissue. We substituted the C-5 nitrile group of S-Cit with an alkynyl group and synthesized Alk-S-Cit. Computational simulations showed that the binding orientation of the labeled drug differed only minimally from that of the original, while analyses performed using LC-MS/MS and microdialysis HPLC confirmed that the incorporation of the alkyne tag affected neither the brain transitivity of the synthesized drug nor its ability to inhibit serotonin reuptake in the brain. The proposed SERS method, when used with silver nanoparticles, was shown to be effective at detecting Alk-S-Cit at the nano-molar level, and we successfully detected alkyne signals in the dorsal raphe nucleus of the mouse brain. Finally, by combining SERS imaging with fluorescence microscopy, we serially imaged Alk-S-Cit and fluorescently labeled neuronal membranes or cerebral blood vessels. In doing so, we were able to reveal the localization of Alk-S-Cit relative to neuronal membranes and the vessel-parenchyma distribution of Alk-S-Cit. Our results show that our multimodal imaging method is a promising tool for directly detecting alkyne-tagged low-molecular weight compounds in tissues and has high potential for elucidating the distribution of drugs together with other biological components.

Being able to image the distribution of SSRIs directly may provide critical insights regarding their underlying mechanisms of the main and side effects. It is widely accepted that SSRIs take several weeks to have a clinical effect, and their precise mechanism of action remains unclear (32, 33). SSRIs act mainly on SERT but also bind to other monoamine transporters and targets, albeit with lower affinities (34). SSRIs are also reported to have clinically relevant actions independent of SERT, such as by activating tropomyosin receptor kinase B (TrkB), a receptor for the brain-derived neurotrophic factor (35). Previous PET studies utilized radio-labeled ligands to the SERT, and their results depended on regional SERT expression, as well as the binding affinities of radio-labeled ligands with respect to SERT $(36,37)$. MSI is an alternative option for detecting low-molecular weight compounds directly without labeling at a higher spatial resolution. However, the ionization of both low-molecular weight compounds and proteins from the tissue, which is required for determining the precise location of a molecule relative to blood vessels within the target view, had been challenging. Our proposed method overcomes such limitations, and allows the direct detection of drugs regardless of the target protein and in their original place at the micrometer scale. Furthermore, the compatibility of the system with fluorescence imaging allows the visualization of fluorescently labeled molecules other than the alkyne-labeled drug. We tested our system using brain samples, but it can also be applied to other peripheral tissues. Therefore, our multimodal method can potentially yield distribution patterns that are different from currently available methods and contribute to the understanding of the mechanisms of the main and side effects of drugs through the examination of CNS and peripheral tissues.

The alkyne SERS peaks detected in the brain sections were consistently observed at approximately 2170 $\mathrm{cm}^{-1}$, with peak shifts spanning from $2118 \mathrm{~cm}^{-1}$ to $2180 \mathrm{~cm}^{-1}$ (Figure $4 \mathrm{G}$ and Figure 5, B and G). In addition, we detected multiple peaks from the same hotspot (Figure $4 \mathrm{G} ; 2118 \mathrm{~cm}^{-1}$ and $2141 \mathrm{~cm}^{-1}$, in spectrum $\mathrm{f}$ ). At low concentrations, such as the single-molecule level, the relative orientation of the molecules against the metal nanoparticles may cause a significant shift in the SERS peaks (38). Our data from the LC-MS/MS experiments and SERS imaging suggest that Alk-S-Cit concentrations in the brain tissue were indeed in the nano-molar levels (Figure 2D, Figure 4, and Figure 5). Thus, the observed peak shifts are likely caused by the differences in the molecular conditions induced by the different dipole moments at low molecular concentrations.

In addition to being a Raman tag, the alkynyl group is also used commonly as a bioorthogonal tag that can be detected readily by click chemistry. However, we believe our SERS method would be more suitable than click chemistry for detecting low-molecular weight compounds in brain tissue for the following reasons. The main binding site of SERT is located halfway down the cell membrane, and one must use reagents (azide probe and catalyst copper) to access the accessible surface area and reach the alkyne group, which has been determined to exist $10 \AA(1 \mathrm{~nm})$ from the surface. Despite the relatively small size of azide fluorescent probes, access to the alkyne group may be hindered by various interactions. Our data support 

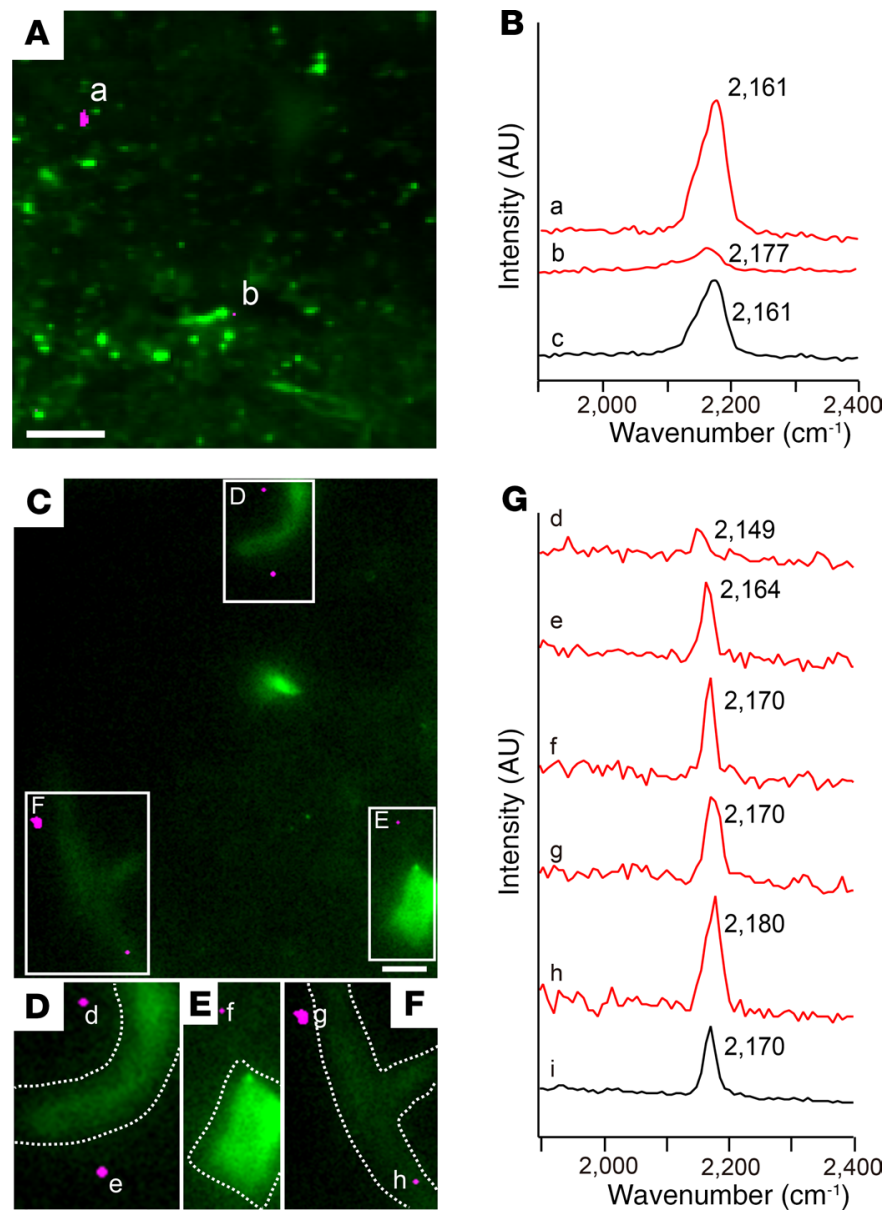

Figure 5. Multimodal SERS and fluorescence imaging of Alk-S-Cit and fluorescently labeled biological components. (A) Representative merged fluorescence image of neuronal membranes (green, GFP) and SERS distribution (magenta) representing signal at $2170 \mathrm{~cm}^{-1}$ of Alk-S-Cit on the dorsal raphe. All images acquired from the same section. Scale bar: $10 \mu \mathrm{m}$. (B) Spectrums a and b show SERS spectra at positions shown in $\mathbf{A}$, and spectrum c shows average of these spectra. (C) Representative merged fluorescence image of cerebral blood vessels (green, DyLight 488) and SERS distribution (magenta) representing signal at 2170 $\mathrm{cm}^{-1}$ of Alk-S-Cit on the dorsal raphe. (D-F) Magnified images of areas outlined by white boxes in C. Scale bar: $10 \mu \mathrm{m}$. (G) Spectrums d-h show SERS spectra at positions shown in $\mathbf{D}-\mathbf{F}$, and spectrum i shows average of these spectra.

this conclusion, as the alkyne SERS peaks were observed at approximately $2170 \mathrm{~cm}^{-1}$ (Figure $4 \mathrm{G}$ and Figure 5, B and G), suggesting that there had been no direct adsorption of Alk-S-Cit onto the surfaces of the silver nanoparticles. This would confirm that accessing the binding pocket would, indeed, be difficult. The SERS signal can be enhanced up to $2.8 \mathrm{~nm}$ away from the intended target (39), meaning that this method can detect drugs inaccessible by click reactions. Secondly, although click reactions are relatively faster (reactions can be completed in minutes, depending on compound and catalyst used) than other biological reactions (40), tissue exposure to the reaction mixture would result in the diffusion of Alk-S-Cit as it is not covalently bound to its target, leading to a loss of both the drug and the related spatial information. Therefore, our approach of using SERS to detect the low-molecular weight drugs in brain tissue is more suitable compared with click chemistry.

While we succeeded in detecting Alk-S-Cit from the dorsal raphe, it remains unclear whether or not the compound was bound to SERT. In an attempt to distinguish SERT-bound Alk-S-Cit from those not bound, we synthesized a previously reported compound, coined benzophenone-alkyne-tagged S-Cit (Benz-AlkS-Cit) for convenience (ref. 41 and Supplemental Figures 7-10). Benz-Alk-S-Cit possesses the backbone structure of both S-Cit and an alkynyl group but shows a very low binding affinity toward SERT. However, the compound exhibited a considerably low transitivity to the brain; thus, it was not suitable for subsequent SERS imaging (Supplemental Figure 11). Our acquired data of alkyne SERS peaks from Alk-S-Cit were observed at the wavelength attributed to the alkyne tag in close proximity to the silver nanoparticle and, thus, suggested Alk-S-Cit was bound to a target and directly inaccessible for the nanoparticle. Alk-S-Cit was simulated to bind to SERT in a similar orientation to S-Cit (Figure 1) and exhibited SERT-inhibiting properties as proven by an increase in extracellular serotonin levels in vivo (Figure 3). Furthermore, distinct alkyne SERS signals were detected from the dorsal raphe, an area with high SERT expression; however, no significant peaks were observed from the dorsomedial periaqueductal gray, an area with low SERT expression (Supplemental Figure 6). This may possibly reflect the differing expression levels of SERT between the 
brain regions and the accumulation of Alk-S-Cit in SERT-expressing areas. While it is tempting to draw the conclusion that the detected Alk-S-Cit was bound to SERT, we cannot rule out the possibility that Alk$\mathrm{S}$-Cit was bound to proteins other than SERT. Further quantitative analyses across multiple brain regions combined with target proteins tagged to genetically encoded fluorescence molecules will be required to confirm this speculation.

While we could directly detect Alk-S-Cit at a high spatial resolution, our study has several limitations. Incorporating an alkyne group within the structure of the target compound at a detectable position while ensuring that there are no changes in the pharmacological action and pharmacokinetics of the compound is a demanding and time-consuming process. Furthermore, extending the applicability of the proposed method to a wide range of drugs may prove challenging. The synthesis of regularly shaped silver nanoparticles may also have a determining effect on the degree of signal enhancement and the quantitative results obtained. The distribution of the nanoparticles across the tissue surface and to its deeper layers will influence the detection probability of the target. Additionally, competitive binding by biological molecules to the nanoparticle surface may hinder the probability of the desired compound being detected. The development of an improved method of applying the nanoparticles onto the tissue may help overcome such challenges and allow for accurate quantitative comparisons between different samples.

In conclusion, we were able to demonstrate the direct in situ detection of a low-molecular weight compound in brain tissue by SERS at a high spatial resolution by using a small chemical tag. Our approach of combining SERS with fluorescence microscopy can potentially provide new information regarding the niche brain areas to which a medicinal compound is delivered and help elucidate the underlying mechanisms of CNS targeting drugs, including SSRIs.

\section{Methods}

Animals. Male C57BL/6 mice 8-12 weeks in age (SLC) were used for the experiments. The mice were subjected to a 12-hour light/dark cycle (lights on from 8 a.m.) and kept in a room with controlled temperature, where water and food (CMF, Oriental Yeast) were available ad libitum.

General organic synthesis. (S)-citalopram oxalate, or (+)-(S)-1-[3-(Dimethylamino)propyl]-1-(4-fluorophenyl)-1,3-dihydro-5-isobenzofurancarbonitrile oxalate, was purchased from Tocris Bioscience. All the reagents and solvents were used as purchased without further purification. All the reactions were performed in an $\mathrm{N}_{2}$ atmosphere. Silica gel (40-100 $\mu \mathrm{m}$; Kanto Chemical Co. Inc.) and precoated TLC plates ( $60 \mathrm{~F}_{254}$; Merck) were used for column chromatography and TLC. The spots on the TLC plates were detected by spraying an acidic $p$-anisaldehyde solution ( $p$-anisaldehyde, $25 \mathrm{~mL} ; c-\mathrm{H}_{2} \mathrm{SO}_{4}, 25 \mathrm{~mL}$; AcOH, $5 \mathrm{~mL}$; EtOH, $425 \mathrm{~mL}$ ) on the plates and then heating them.

Synthesis of Alk-S-Cit and Benz-Alk-S-Cit. $\mathrm{PtO}_{2}(8.0 \mathrm{mg})$ was added to a solution of (S)-citalopram oxalate $(83.0 \mathrm{mg}, 0.20 \mathrm{mmol})$ in $80 \% \mathrm{HCOOH}(1.0 \mathrm{~mL})$, and the mixture was stirred at $75^{\circ} \mathrm{C}$ for 6 hours. The reaction mixture was then filtered through a cotton pad, and the filtrate was basified with a saturated aqueous solution of $\mathrm{NaHCO}_{3}$. The resulting solution was then extracted using AcOEt $(2 \times 20 \mathrm{~mL})$, and the combined AcOEt extract was dried over $\mathrm{Na}_{2} \mathrm{SO}_{4}$. The solvent was removed from the AcOEt extract under reduced pressure, yielding formylated $(S)$-citalopram (S-Cit), which was used in the next reaction without further purification. We added $\mathrm{K}_{2} \mathrm{CO}_{3}(82.9 \mathrm{mg}, 0.6 \mathrm{mmol})$ and dimethyl (1-diazo-2-oxopropyl) phosphonate $(45.0 \mu \mathrm{L}, 57.6 \mathrm{mg}, 0.30 \mathrm{mmol})$ to a solution of the above product in $\mathrm{MeOH}(2.0 \mathrm{~mL})$, and the mixture was stirred at room temperature for 3 hours. The reaction was quenched by adding $\mathrm{H}_{2} \mathrm{O}$ to the reaction flask, and the mixture was extracted with $\operatorname{AcOEt}(2 \times 20 \mathrm{~mL})$. The combined AcOEt extract was dried over $\mathrm{Na}_{2} \mathrm{SO}_{4}$ and was concentrated in vacuo. The crude product was purified by $\mathrm{SiO}_{2}$ column chromatography $\left(\mathrm{CHCl}_{3} / \mathrm{MeOH} / \mathrm{H}_{2} \mathrm{O}=60: 3: 1\right.$, lower phase $)$ to obtain the desired compound - namely, alkynylated S-Cit (35.5 mg, 55\% in 2 steps) - as a colorless oil.

Benz-Alk-S-Cit. $N, N$-diisopropylethylamine $(10 \mu \mathrm{L}, 56.8 \mu \mathrm{mol})$ and mesylate $(9.8 \mathrm{mg}, 28.4 \mu \mathrm{mol})$ were added to a solution of $(S)$-desmethylcitalopram $(8.9 \mathrm{mg}, 28.6 \mu \mathrm{mol})$ in $\mathrm{MeCN}(300 \mu \mathrm{L})$, and the whole mixture was stirred at room temperature for 13 hours. The reaction mixture was quenched by adding saturated $\mathrm{NH}_{4} \mathrm{Cl}$ aqueous to the flask, and the whole mixture was extracted with AcOEt $(3 \times 1 \mathrm{~mL})$. The combined AcOEt extract was dried over $\mathrm{Na}_{2} \mathrm{SO}_{4}$ and was concentrated in vacuo. The crude product was purified by $\mathrm{SiO}_{2}$ preparative TLC $\left(\mathrm{CH}_{2} \mathrm{Cl}_{2} / \mathrm{MeOH}=20: 1\right)$ to provide the desired Benz-Alk-S-Cit $(7.1 \mathrm{mg}, 44 \%)$ as a colorless oil.

Spectroscopic analysis. The NMR spectra were measured using an AL-400 system $\left({ }^{1} \mathrm{H}, 400 \mathrm{MHz} ;{ }^{13} \mathrm{C}, 100\right.$ $\mathrm{MHz}$ (JEOL Ltd.) or a Varian INOVA $600\left({ }^{1} \mathrm{H}, 600 \mathrm{MHz} ;{ }^{13} \mathrm{C}, 150 \mathrm{MHz}\right.$ (Agilent); the tetramethylsilane 
(TMS) or residual solvent $\left(\mathrm{CDCl}_{3}\right)$ peaks were used as the references. For the acquisition of accurate mass, ESI-TOF-MS was performed using a Q-Tof Ultima API mass spectrometer (Waters Co.). MALDI-TOFMS was recorded using a JMS-S3000 (JEOL Ltd.).

Injection of Alk-S-Cit and S-Cit in mice. For the LC-MS/MS quantification process, the mice were i.p. injected with a $5.0 \mathrm{mg} / \mathrm{kg}$ combined dose of Alk-S-Cit and S-Cit dissolved in DMSO. For brain tissue imaging, the mice were i.p. injected with $20 \mathrm{mg} / \mathrm{kg}$ of Alk-S-Cit or S-Cit.

Virus production and purification. The AAV plasmid for viral experiments, pAAV-Syn-Chronos-GFP, was a gift from Edward Boyden (Massachusetts Institute of Technology, Cambridge, Massachusetts, USA) (30) (Addgene plasmid 59170; Addgene). Chronos is a general-use channelrhodopsin that translocates to the cell membrane, which allows the labeling of axons and synaptic terminals. AAVs were generated by triple transfection of Lenti-X 293T cells with AAV helper plasmid (VPK-400-DJ; Cell Biolabs Inc.), AAV-PHP.eB cap plasmid, and the pAAV-Syn-Chronos-GFP plasmid using polyethylenimine (Polysciences Inc.). Viral particles were harvested from the medium 72 hours after transfection and from the cells and the medium 120 hours after transfection. Viral particles from the medium were precipitated for 2 hour on ice, using $40 \%$ polyethylene glycol at a final concentration of $8 \%$. The cell pellets were resuspended in gradient buffer (10 $\mathrm{mM}$ Tris, $150 \mathrm{mM} \mathrm{NaCl}, 10 \mathrm{mM} \mathrm{MgCl}$ ), freeze-thawed 3 times, and then treated with $\geq 250 \mu \mathrm{L}$ Benzonase Nuclease (E1014; MilliporeSigma) at $37^{\circ} \mathrm{C}$ for 1 hour. The viral media were clarified by centrifugation at $4000 \mathrm{~g}$ for 30 minutes at $4^{\circ} \mathrm{C}$, and the resulting pellets were suspended in gradient buffer. This suspension was combined with the suspension from cell pellets and further treated with Benzonase Nuclease for 10 minutes at $4^{\circ} \mathrm{C}$. The cell lysates were clarified by centrifugation at $4000 \mathrm{~g}$ for 30 minutes at $4^{\circ} \mathrm{C}$. The supernatant was then purified over iodixanol (Optiprep; Cosmo Bio Co.) step gradients (15\%, 25\%, 40\%, and 58\%). The viruses were then concentrated using Amicon filters (UFC910024, MilliporeSigma) and formulated in PBS. Virus titers were determined by measuring the number of viral genomes using quantitative PCR with linearized genome plasmid as a standard.

Fluorescent labeling. For neuronal axon labeling, the AAV-PHP.eB-Syn-Chronos-GFP vector was injected with a viral titer of $1 \times 10^{12}$ particles $/ \mathrm{mL}$. At least 3 weeks after AAV injection, brain tissues were collected.

For endothelial staining, DyLight 488-labeled tomato lectin is an effective marker of blood vessels. For Alk-S-Cit and blood vessel imaging, endothelial cells were labeled by the transcardial perfusion of DyLight 488-labeled tomato lectin (catalog DL-1174, Vector Laboratories) as previously described (31).

Tissue preparation. One hour after the injection of Alk-S-Cit or S-Cit, the mice were deeply anesthetized via an i.p. injection of the following combination of anesthetics: $0.3 \mathrm{mg} / \mathrm{kg}$ of medetomidine (Nippon Zenyaku Kogyo Co. Ltd.), $4.0 \mathrm{mg} / \mathrm{kg}$ of midazolam (Sandoz K.K.), and $5.0 \mathrm{mg} / \mathrm{kg}$ of butorphanol (Meiji Seika Pharma Co. Ltd.). Subsequently, the mice were transcardially perfused with saline, which was followed by $4 \%$ paraformaldehyde (PFA) dissolved in PBS. The brains were retrieved and postfixed in $4 \%$ PFA in PBS overnight.

For the LC-MS/MS analysis, $0.5 \mathrm{~mm}$-thick coronal slices were cut using a stainless mouse brain matrix. Sections $2.5-3.0 \mathrm{~mm}$ and $5.5-6.0 \mathrm{~mm}$ posterior to the olfactory bulb were used for quantification. To extract Alk-S-Cit and S-Cit from the sections, $200 \mu \mathrm{L}$ acetonitrile was added to the sections, which were then homogenized using a handheld homogenizer (Physcotron NS-310E3, Microtec Co.). The samples were then centrifuged at 21,900 $\mathrm{g}$ for 5 minutes at room temperature, and $60 \mu \mathrm{L}$ of the supernatant was collected. Next, $60 \mu \mathrm{L}$ of deionized water was added to the supernatant, and the samples were centrifuged again at 21,900 $\mathrm{g}$ for 5 minutes. Alk-S-Cit and S-Cit standards with concentrations of $0-300 \mathrm{mg} / \mathrm{mL}$ were prepared using the supernatants of the brains of the control mice.

For SERS imaging, the fixed brains were embedded with OCT compound (Sakura Finetek Japan Co. Ltd.), and acute sections were cut at $20 \mu \mathrm{m}$ using a cryostat (CM1520; Leica Camera AG). The sections were mounted directly onto Matsunami Adhesive Silane-coated (MAS-coated) glass slides (Matsunami Glass Ind. Ltd.) and stored at $-80^{\circ} \mathrm{C}$ until use.

LC-MS/MS analysis. The LC-MS/MS analyses were carried out using a Waters ACQUITY UPLC system (Waters Co.) interfaced with a Quattro Premier XE triple quadrupole mass spectrometer (Waters Co.). The LC separations were performed using an ACQUITY UPLC BEH C18 column ( $3 \mathrm{~mm} \times 100 \mathrm{~mm}$, $1.7 \mu \mathrm{m}$; Waters Co.) at $40^{\circ} \mathrm{C}$. Gradient elution was performed using a binary gradient of solvent $\mathrm{A}(0.1 \%$ formic acid in deionized water) and solvent B (acetonitrile) as follows: 40\% B from 0-0.7 minutes, $60 \%$ B up to 2.5 minutes, $100 \% \mathrm{~B}$ from 3.0-6.0 minutes, and back to $40 \% \mathrm{~B}$ for $6.5-9.5$ minutes. The flow rate was set to $0.4 \mathrm{~mL} / \mathrm{min}$. A total of $10 \mu \mathrm{L}$ of the sample fluid was injected for analysis. MS/MS was performed 
using an electrospray ionization source in the positive mode. The capillary voltage was set at $4.5 \mathrm{kV}$. Multiple reaction monitoring (MRM) transitions, along with the parameters for Alk-S-Cit and S-Cit, are listed in Supplemental Table 1. The dwell time for each MRM transition was set at 0.1 second. Device control and data acquisition were performed using the software MassLynx 4.1 (Waters Co.). The Alk-S-Cit and S-Cit levels were quantified by analyzing the peak areas of the respective standards.

In silico modeling. Molecular simulations were performed using MacroModel, which was implemented in Maestro Version 11.2 (Schrödinger LLC). Only SERT and S-Cit were used in the S-Cit/SERT complex structure (PDB ID: 5I71). The SERT protein model was prepared using the Protein Preparation Wizard implemented in Maestro. After the replacement of the cyano group in S-Cit with the alkyne group, the complex structure was energy minimized using MacroModel with the optimized potentials for liquid simulations 3 force field, as provided.

Microdialysis. The mice were anesthetized with isoflurane and stereotaxically implanted with a $4 \mathrm{~mm}-$ long guide cannula for a dialysis probe (Eicom Corporation) in the medial prefrontal cortex (Anterior +1.9 $\mathrm{mm}$, Lateral $\pm 0.5 \mathrm{~mm}$, Ventral $-0.8 \mathrm{~mm}$, from the bregma). The guide cannula was cemented with dental acrylic, and postoperation analgesia was performed with an i.p. injection of $0.1 \mathrm{mg} / \mathrm{kg}$ buprenorphine. The experiments were conducted 2 days after surgery. For the microdialysate measurements, a 3-mm active probe membrane was inserted into the guide cannula and perfused with Ringer's solution at a constant flow rate of $1 \mu \mathrm{L} / \mathrm{min}$. The microdialysates were collected every 20 minutes and immediately injected into an HPLC-electrochemical detector system (HTEC-500; Eicom Corporation). Chromatographic separation was performed using an EICOMPAK CAX column (2.0 mm i.d. $\times 200 \mathrm{~mm}$; Eicom Corporation); the mobile phase consisted of $100 \mathrm{mM}$ ammonium acetate buffer (pH 6.0), $30 \mathrm{mM}$ sodium sulfate, $134 \mu \mathrm{M}$ EDTA, and 30\% (vol/ vol) methanol. The potential of the graphite electrode (Eicom Corporation) was set to $+450 \mathrm{mV}$ (versus $\mathrm{Ag} /$ $\mathrm{AgCl}$ reference electrode). The data analyses were performed using Power Chrom (eDAQ Pty Ltd.), while the serotonin concentrations were determined by analyzing the peak area of an external standard.

Nanoparticle synthesis. Silver nanoparticles $(\varphi=23 \mathrm{~nm})$ were synthesized using the Lee \& Meisel method with minor modifications (42). Briefly, a mixture of $30 \mathrm{~mL}$ of glycerol and $40 \mathrm{~mL}$ of $\mathrm{H}_{2} \mathrm{O}$ in a $100-\mathrm{mL}$ flask was heated in an oil bath at $106^{\circ} \mathrm{C}$ for 30 minutes. Then, $18 \mathrm{mg}$ of silver nitrate dissolved in $1.0 \mathrm{~mL}$ of $\mathrm{H}_{2} \mathrm{O}$ was added to the above mixture under vigorous magnetic stirring. After 1 minute, $2.0 \mathrm{~mL}$ of sodium citrate $(3 \%)$ was added. The solution was stirred for another hour and then cooled to room temperature. The silver nanoparticle dispersion was centrifuged, and the final concentration of the dispersion was approximately $1.6 \times 10^{13}$ particles $/ \mathrm{mL}$. To prepare the nanoparticle-dispersed substrates, $50 \mu \mathrm{L}$ of silver nanoparticle dispersion was incubated for 2 hours on an aminosilane-coated cover glass.

SERS imaging. The brain tissue was sliced into $20 \mu \mathrm{m}$-thick slices, which were sandwiched between a glass slide and the nanoparticle-coated cover glass. The nanoparticle dispersion was drop-cast onto the cover glass before the encapsulation of the brain tissue slice. The substrate was then placed on the sample plane of an inverted microscope (ECRPSE Ti, Nikon Corporation), which was a part of a laboratory-built line-illumination Raman microscopy system. The optical setup has been described elsewhere $(43,44)$. Briefly, a line-shaped laser beam $(\lambda=532 \mathrm{~nm})$ was generated using a cylindrical lens and focused using an objective lens (40×, NA 1.25; Nikon Corporation). The scattered light was collected with an EM-CCD camera (iXon Ultra 888; Andor Technology Ltd.) through the entrance of a spectrophotometer (MK-300; Bunkoukeiki Co. Ltd.). The laser beam was scanned at right angles to the line-shape of the focused beam using a galvanometer mirror, and SERS imaging was performed. The laser power was $0.4 \mathrm{~mW} / \mu \mathrm{m}^{2}$ at the sample plane, and the exposure time was $200 \mathrm{~ms}$ for 1 line scan.

Signal processing of SERS spectra. The wavenumber range of $1830-2340 \mathrm{~cm}^{-1}$ was used for signal processing. The alkyne used exhibits a characteristic peak at approximately $2170 \mathrm{~cm}^{-1}$. Furthermore, the other molecules do not exhibit any peak in the same region $\left(1830-2340 \mathrm{~cm}^{-1}\right)$. Before the polynomial fitting for baseline correction, we excluded the wavenumber region (1984-2226 cm $\mathrm{cm}^{-1}$ ) where the alkyne exhibits a peak, in order to ensure that the alkyne peak did not affect the polynomial fitting results. The polynomial fitting was performed using a second-order polynomial function. The SDs of the baseline-corrected spectra were calculated in order to distinguish the alkyne peak from the background signal. The signal processing was performed using Matlab (The MathWorks Inc.).

Statistics. Statistical analyses were performed using GraphPad Prism 7.04 (GraphPad Software Inc.). The statistical differences were determined using 2-way ANOVA (Figure 2D) and 2-way repeated-measures ANOVA (Figure 3B). 
Study approval. The design of the study, which used laboratory animals, was approved by the Animal Care and Use Committee of Osaka University (approval no. 28-1-12). The animals were handled carefully following the guidelines of the Animal Care and Use Committee of Osaka University.

\section{Author contributions}

A. Kasai and $\mathrm{HH}$ conceived and designed the study; MT, KB, NK, A. Kimishima, and K. Higashino performed experiments with help from K. Harada, MA, YA, KS, and KF; MM performed computational modeling with help KK; SK and KF provided methodology and tools; and MT, KB, A. Kasai, and $\mathrm{HH}$ wrote the manuscript, with input from all coauthors.

\section{Acknowledgments}

We thank Ken-ichi Inoue and Masahiko Takada (Primate Research Institute, Kyoto University, Aichi, Japan) for his donation of the AAV-PHP.eB capsid plasmid. This work was supported in part by JSPS KAKENHI grant nos. JP17H050534 (A. Kasai), JP18K19399 (A. Kasai), JP17H03989 (HH), JP17K19488 (HH), JP19K07121 (HH), JP17H02800 (KF), and JP26000011 (SK); MEXT KAKENHI grant nos. JP18H05132 (A. Kasai), JP19H05217 (A. Kasai), and JP18H05416 (HH); AMED under grant nos. JP19dm0107122 (HH), JP19dm0207061 (HH), JP18jm0610007 (A. Kasai), and JP19am0101084 (HH); grants from the Mochida Memorial Foundation for Medical and Pharmaceutical Research, Japan (A. Kasai), and the Takeda Science Foundation, Japan (A. Kasai and $\mathrm{HH}$ ).

Address correspondence to: Atsushi Kasai or Hitoshi Hashimoto, Graduate School of Pharmaceutical Sciences, Osaka University, 1-6 Yamadaoka, Suita, Osaka 565-0871, Japan. Phone: 81.6.6879.8182; Email: kasai@phs.osaka-u.ac.jp (A. Kasai); hasimoto@phs.osaka-u.ac.jp (HH). Or to: Katsumasa Fujita, Department of Applied Physics, Graduate School of Engineering, Osaka University, 2-1 Yamadaoka, Suita, Osaka 565-0871, Japan. Phone: 81.6.6879.7876; Email: fujita@ap.eng.osaka-u.ac.jp.

1. Genon S, Reid A, Langner R, Amunts K, Eickhoff SB. How to Characterize the Function of a Brain Region. Trends Cogn Sci (Regul Ed). 2018;22(4):350-364.

2. Glasser MF, et al. A multi-modal parcellation of human cerebral cortex. Nature. 2016;536(7615):171-178

3. Lundberg J, Christophersen JS, Petersen KB, Loft H, Halldin C, Farde L. PET measurement of serotonin transporter occupancy: a comparison of escitalopram and citalopram. Int J Neuropsychopharmacol. 2007;10(6):777-785.

4. Baldinger $\mathrm{P}$, et al. Regional differences in SERT occupancy after acute and prolonged SSRI intake investigated by brain PET. Neuroimage. 2014;88:252-262.

5. Kim E, et al. Regional Differences in Serotonin Transporter Occupancy by Escitalopram: An $\left[{ }^{11}\right.$ C]DASB PK-PD Study. Clin Pharmacokinet. 2017;56(4):371-381.

6. Vismeh R, Waldon DJ, Teffera Y, Zhao Z. Localization and quantification of drugs in animal tissues by use of desorption electrospray ionization mass spectrometry imaging. Anal Chem. 2012;84(12):5439-5445.

7. Shariatgorji M, et al. Simultaneous imaging of multiple neurotransmitters and neuroactive substances in the brain by desorption electrospray ionization mass spectrometry. Neuroimage. 2016;136:129-138.

8. Wei L, et al. Super-multiplex vibrational imaging. Nature. 2017;544(7651):465-470

9. Taylor J, Huefner A, Li L, Wingfield J, Mahajan S. Nanoparticles and intracellular applications of surface-enhanced Raman spectroscopy. Analyst. 2016;141(17):5037-5055.

10. Laing S, Jamieson LE, Faulds K, Graham D. Surface-enhanced Raman spectroscopy for in vivo biosensing. Nat Rev Chem. 2017;1(13):0060.

11. Yuen C, Zheng W, Huang Z. Surface-enhanced raman scattering: principles, nanostructures, fabrications, and biomedical applications. J Innov Opt Health Sci. 2008;01(02):267-284.

12. Hossain MK, Kitahama Y, Huang GG, Han X, Ozaki Y. Surface-enhanced Raman scattering: realization of localized surface plasmon resonance using unique substrates and methods. Anal Bioanal Chem. 2009;394(7):1747-1760.

13. Ando J, et al. Alkyne-Tag SERS Screening and Identification of Small-Molecule-Binding Sites in Protein. J Am Chem Soc. 2016;138(42):13901-13910.

14. Yamakoshi $\mathrm{H}$, et al. Alkyne-tag Raman imaging for visualization of mobile small molecules in live cells. J Am Chem Soc. 2012;134(51):20681-20689.

15. Hong S, Chen T, Zhu Y, Li A, Huang Y, Chen X. Live-cell stimulated Raman scattering imaging of alkyne-tagged biomolecules. Angew Chem Int Ed Engl. 2014;53(23):5827-5831.

16. Yamakoshi $\mathrm{H}$, et al. Imaging of EdU, an alkyne-tagged cell proliferation probe, by Raman microscopy. J Am Chem Soc 2011;133(16):6102-6105.

17. Zhang P, et al. Structure-activity relationships for a novel series of citalopram (1-(3-(dimethylamino)propyl)-1-(4-fluorophenyl)-1,3-dihydroisobenzofuran-5-carbonitrile) analogues at monoamine transporters. J Med Chem. 2010;53(16):6112-6121.

18. Banala AK, et al. Design and synthesis of 1-(3-(dimethylamino)propyl)-1-(4-fluorophenyl)-1,3-dihydroisobenzofuran-5-carbonitrile (citalopram) analogues as novel probes for the serotonin transporter S1 and S2 binding sites. JMed Chem. 
2013;56(23):9709-9724.

19. Kumar V, Rahbek-Clemmensen T, Billesbølle CB, Jorgensen TN, Gether U, Newman AH. Novel and high affinity fluorescent ligands for the serotonin transporter based on (s)-citalopram. ACS Med Chem Lett. 2014;5(6):696-699.

20. Klebe G. Applying thermodynamic profiling in lead finding and optimization. Nat Rev Drug Discov. 2015;14(2):95-110.

21. Bræstrup C, Sanchez C. Escitalopram: a unique mechanism of action. Int J Psychiatry Clin Pract. 2004;8 Suppl 1:11-13.

22. Coleman JA, Green EM, Gouaux E. X-ray structures and mechanism of the human serotonin transporter. Nature. 2016;532(7599):334-339.

23. Kobayashi H, Ogawa M, Alford R, Choyke PL, Urano Y. New strategies for fluorescent probe design in medical diagnostic imaging. Chem Rev. 2010;110(5):2620-2640.

24. Kato T, et al. Sestrin modulator NV-5138 produces rapid antidepressant effects via direct mTORC1 activation. J Clin Invest. 2019;129(6):2542-2554

25. Beyer CE, Cremers TI. Do selective serotonin reuptake inhibitors acutely increase frontal cortex levels of serotonin? Eur J Pharmacol. 2008;580(3):350-354.

26. Zhang Z, Bando K, Mochizuki K, Taguchi A, Fujita K, Kawata S. Quantitative Evaluation of Surface-Enhanced Raman Scattering Nanoparticles for Intracellular pH Sensing at a Single Particle Level. Anal Chem. 2019;91(5):3254-3262.

27. Lim JK, Joo SW, Shin KS. Concentration dependent Raman study of 1,4-diethynylbenzene on gold nanoparticle surfaces. Vib Spectrosc. 2007;43(2):330-334.

28. Kennedy DC, McKay CS, Tay LL, Rouleau Y, Pezacki JP. Carbon-bonded silver nanoparticles: alkyne-functionalized ligands for SERS imaging of mammalian cells. Chem Commun (Camb). 2011;47(11):3156-3158.

29. Lein ES, et al. Genome-wide atlas of gene expression in the adult mouse brain. Nature. 2007;445(7124):168-176.

30. Klapoetke NC, et al. Independent optical excitation of distinct neural populations. Nat Methods. 2014;11(3):338-346.

31. Jährling N, Becker K, Dodt HU. 3D-reconstruction of blood vessels by ultramicroscopy. Organogenesis. 2009;5(4):227-230.

32. Krishnan V, Nestler EJ. The molecular neurobiology of depression. Nature. 2008;455(7215):894-902.

33. Insel TR. Disruptive insights in psychiatry: transforming a clinical discipline. J Clin Invest. 2009;119(4):700-705.

34. Sanchez C, Reines EH, Montgomery SA. A comparative review of escitalopram, paroxetine, and sertraline: Are they all alike? Int Clin Psychopharmacol. 2014;29(4):185-196.

35. Rantamäki T, et al. Antidepressant drugs transactivate TrkB neurotrophin receptors in the adult rodent brain independently of BDNF and monoamine transporter blockade. PLoS One. 2011;6(6):e20567.

36. Laruelle M, Slifstein M, Huang Y. Relationships between radiotracer properties and image quality in molecular imaging of the brain with positron emission tomography. Mol Imaging Biol. 2003;5(6):363-375.

37. Bengel D, et al. Cellular localization and expression of the serotonin transporter in mouse brain. Brain Res. 1997;778(2):338-345.

38. Etchegoin PG, Le Ru EC. Resolving single molecules in surface-enhanced Raman scattering within the inhomogeneous broadening of Raman peaks. Anal Chem. 2010;82(7):2888-2892.

39. Stiles PL, Dieringer JA, Shah NC, Van Duyne RP. Surface-enhanced Raman spectroscopy. Annu Rev Anal Chem (Palo Alto Calif). 2008;1:601-626.

40. Haldón E, Nicasio MC, Pérez PJ. Copper-catalysed azide-alkyne cycloadditions (CuAAC): an update. Org Biomol Chem. 2015;13(37):9528-9550.

41. Yarravarapu N, Geffert L, Surratt CK, Cascio M, Lapinsky DJ. Clickable photoaffinity ligands for the human serotonin transporter based on the selective serotonin reuptake inhibitor (S)-citalopram. Bioorg Med Chem Lett. 2018;28(21):3431-3435.

42. Steinigeweg D, Schlücker S. Monodispersity and size control in the synthesis of 20-100 nm quasi-spherical silver nanoparticles by citrate and ascorbic acid reduction in glycerol-water mixtures. Chem Commun (Camb). 2012;48(69):8682-8684.

43. Hamada K, Fujita K, Smith NI, Kobayashi M, Inouye Y, Kawata S. Raman microscopy for dynamic molecular imaging of living cells. J Biomed Opt. 2008;13(4):044027.

44. Palonpon AF, et al. Raman and SERS microscopy for molecular imaging of live cells. Nat Protoc. 2013;8(4):677-692. 